\title{
A NOVEL BIO-WASTE INCORPORATED ALGINATE SORBENT FOR DE-FLUORIDATION OF WATER
}

\author{
Carmalin Sophia Ayyappan', S. Sreeja ${ }^{2}$, V.M. Bhalambaal' ${ }^{1}$ \\ 1 National Environmental Engineering Research Institute, CSIR Complex, Taramani, Chennai 600113, India, \\ e-mail: ac_sophia@neeri.res.in \\ 2 Academy of Scientific and Innovative Research, Taramani, Chennai 600113, India
}

Received: 2015.03 .15

Accepted: 2015.08.31

Published: 2015.10.01

\begin{abstract}
This study evaluates the feasibility of using tamarind (Tamarindus indica) seed powder for de-fluoridation of fluoride contaminated water. Batch study confirmed that tamarind seeds in dry powder form could remove $87 \%$ of fluoride from water. This bio-sorbent can be used effectively in areas where fluoride concentrations are above the permissible limits of $1.5 \mathrm{mg} \cdot \mathrm{l}^{-1}$ as per WHO Standard, 1984. Tamarind seed powder was incorporated in a matrix of sodium alginate and made into gel-beads. The beads were tested for de-fluoridation efficiency by conducting column studies. The effect of various factors, such as flow rate, retention time, and the number of runs on the efficacy of fluoride removal was also studied. The results revealed that flow rate did not seem to have much effect on the percentage fluoride removal but the fluoride concentration decreased drastically upon greater retention time and multiple runs.
\end{abstract}

Keywords: tamarind seeds, de-fluoridation, sodium alginate, column study, water purification.

\section{INTRODUCTION}

Water is one of the most abundant and crucial components of our life system, but today most of the countries have scarcity of clean water. Water pollution is one of the serious problems in India. Poor drinking water quality contributes to $80 \%$ of the diseases in the world [WHO 1984] and 65\% of endemic fluorosis in the world is a result of fluoride contamination in drinking water [Felsenfeld, Robert 1991, WHO 2002].

Many water sources are unsafe for human consumption as well as for other activities, such as irrigation and industrial needs. Potable water is contaminated at many places by various pollutants, such as fluorides, nitrates, iron etc.

Fluoride is widely distributed in soil, water, air, vegetation, agricultural products and sea foods. Ground water sources may contain excess fluoride where minerals like cryolites, biotites and fluoroapatite are present. This is mainly due to the dissolution of fluoride from fluoride bear- ing minerals [Kudesia 2003]. Fluorite $\left(\mathrm{CaF}_{2}\right)$ is a source of industrial mineral and a major source of fluoride contamination in water and soil. The disposal of industrial waste leads to the contamination of river with fluoride along with other toxic materials. Fluoride is essential for normal mineralization of the bones and formation of dental enamel [Ceopalan 2003].

Fluoride, if taken in small quantities, is usually beneficial; on the contrary, long-term consumption of water containing excessive amounts of fluoride can lead to fluorosis of teeth and bones due to replacement of calcium in the bones and teeth. The excessive intake of fluoride may cause dental and skeletal disorders [Jamodei et al. 2004, Gonzales et al. 2004, Solangi et al. 2009].

Reverse osmosis (RO) can completely defluoridate water, but in practice, it depends on the level of pressure applied and membrane capacity [Meenakshi, Maheshwari 2006]. This process is energy intensive and it is not cost-effective. Hence, more techno-economically feasible solu- 
tions i.e., passive filtration methods are required that are not dependent on electricity. These methods should use inexpensive materials and simple treatment procedures, which can be maintained in rural areas at low costs.

Hence, there is an imminent need of alternative passive de-fluoridation solutions. Different biomaterials have been used for purification of water since ancient times [Ige et al. 2012]. Studies have reported the use of biomaterials, such as tamarind seed [Subramanian, Dhana Ramalakshmi 2010] rice husk, egg shell, peanut shells, saw dust, coir dust, sea weeds and corn cobs [Mondal et al. 2012]. An earlier study has proved the ability of tamarind seed kernel powder to remove chromium and other heavy metal ions from by forming complexes through replacement of hydrogen [Mishra 2013]. This seems to be cost effective in the removal of fluoride. This bio-sorbent can replace activated alumina which is the only household solution available currently. However, activated alumna process requires huge quantities of water, chemicals for pre-treatment and regeneration/disposal of used beds.

This study investigates the potential of tamarind seed powder, a waste from food industry for the removal of fluoride ions in water. The tamarind seed powder was immobilized in alginate matrix for easy handling and use. The study was aimed to devise a simple, inexpensive and viable de-fluoridation method that could be adopted easily by rural and semi- urban communities. The factors influencing fluoride removal were studied using a column packed with tamarind-alginate beads. The beads were characterized using Scanning Electron Microscopy (SEM) and EDX.

\section{MATERIALS AND METHODS}

\section{Estimation of fluoride concentration}

SPADNS Method 10225 [APHA, AWWA, WEF 2005] was followed to estimate fluoride concentrations in water. Sodium fluoride $(\mathrm{NaF})$ was used to prepare the synthetic fluoride solution of standard concentrations for SPADNS calibration. Fluoride concentrations in ground water in India vary significantly. In some parts of India, fluoride levels are below $0.5 \mathrm{mg}^{-1}{ }^{-1}$, while in other places, fluoride levels are as high as 30 $\mathrm{mg}^{-1} \mathrm{1}^{-1}$ [Handa 1975]. Synthetic fluoride solution of $2 \mathrm{mg}^{-1}$ was used for all column and batch tests, since the average concentration of fluoride contamination in Andhra Pradesh (India) was $1.996 \pm 0.500 \mathrm{mg}^{-1} \mathrm{1}^{-1}$ [Brindha et al. 2011]. Random field water samples (10 nos.) were collected and tested from largely fluoride contaminated areas, in rural South Andhra Pradesh, India and was found to have fluoride values close to $2 \mathrm{mg}^{-1}{ }^{-1}$.

\section{Preparation of bio-sorbent material}

The tamarind seeds were collected from the local market, washed to remove any contaminants; and air dried at $60{ }^{\circ} \mathrm{C}$ for 2 days. The dried seeds were powdered using a home blender to a particle size of 200-250 micron. 3\% sodium alginate solution was prepared and $2 \mathrm{~g}$ of tamarind seed powder was mixed for making beads. The beads were prepared using a peristaltic pump dripping method into an excess of $0.2 \mathrm{M} \mathrm{CaCl}_{2}$ solution at room temperature. The beads were left in the solution for 3 hours and later rinsed thoroughly several times with distilled water. Figure 1 shows the picture of the beads prepared.

\section{Batch testing of the bio-sorbent}

Preliminary laboratory tests were conducted to check the fluoride removal efficacy. Batch sorption tests were carried out with a known weight of bio-sorbent in a predetermined volume of synthetic fluoride solution. The contents were agitated in a rotary shaker $(120 \mathrm{rpm})$. After 2 hours of contact time, the sorbent was separated and fluoride concentration was estimated in the filtrate.

\section{Fixed bed column set-up}

The filter column of $2.5 \mathrm{~cm}$ diameter was loaded with the adsorbent to a height of $60 \mathrm{~cm} .50$ $\mathrm{ml}$ of standard solution of fluoride $\left(2 \mathrm{mg}^{-1}\right)$ was run through the filter column during the experiments. The filtrate was estimated for residual fluoride concentration by SPADNS Method [APHA, AWWA, WEF 2005]. The fixed bed column is shown in Figure 1.

\section{Analytical techniques}

Scanning electron microscopy was done on select samples using Hitachi S-520 SEM instrument at an accelerating voltage of $15 \mathrm{kV}$ and Hitachi HUS 5GB vacuum evaporator for gold coating. UV-Vis Spectrophotometer (Shimadzu) was used at an absorption wavelength of $570 \mathrm{~nm}$ 


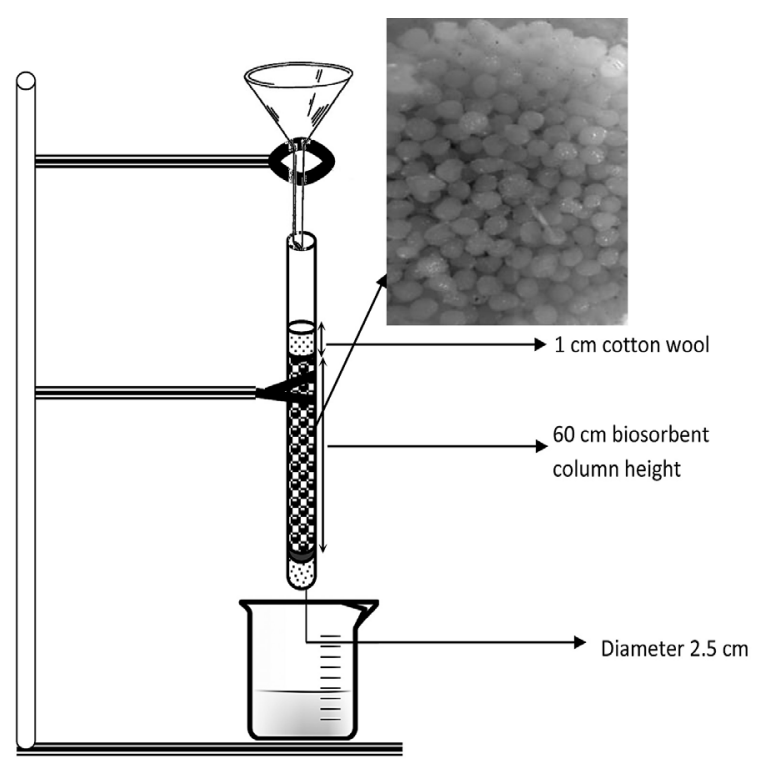

Figure 1. Fixed bed column set up

to measure the fluoride concentrations before and after each run. The analysis and runs were in triplicate for all the experiments.

The samples have been scanned in a Nicolet 6700 , Thermo-Scientific instrument over the MidIR Region from $4500-400 \mathrm{~cm}^{-1}$. The spectrum obtained is averaged for 36 times with a spectral resolution of $4 \mathrm{~cm}^{-1}$.
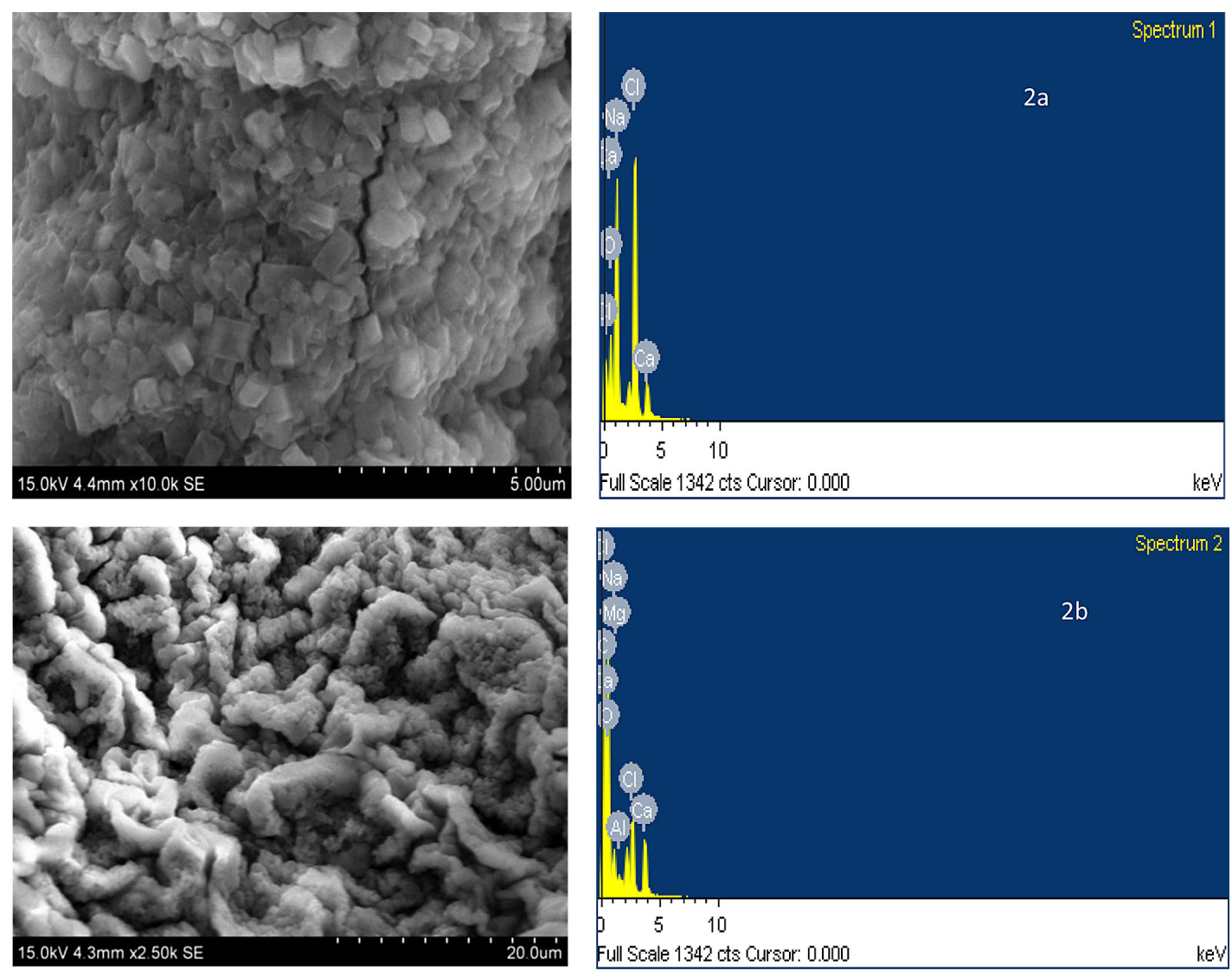

Figure 2. $a$ - SEM and EDX of the control beads, $b$ - SEM and EDX of the tamarind-alginate beads
BET Analysis for measuring the pore size and, pore volume and pore size distribution was performed using Quantachrome Instuments ASiQwin - Automated Gas Sorption Data Acquisition and Reduction.

\section{RESULTS AND DISCUSSION}

\section{Characterization of adsorbent biomaterials Scanning electron microscopy}

Scanning Electron Microscopy (SEM) images of the bio-sorbent i.e., the control alginate beads (with no tamarind seed powder) and tamarind seed powder incorporated alginate beads were taken at several magnifications to observe the morphological changes due to the immobilization of tamarind seed powder. The SEMEDX of the same was shown in Figure 2a and Figure $2 b$ respectively. Images of the control beads show cubical structures, whereas that of the tamarind-alginate bio-sorbent bead appears to be heterogenic likely due to the organic components in the tamarind seed powder. The EDX confirms the presence of organic components in the tamarind. The organic functional groups 
may play a vital role in the absorption of fluoride. This could complement the innate fluoride removal property of tamarind seed powder. Hence, the combination is synergistic. EDX of the tamarind-alginate beads also shows the presence of magnesium, and aluminium which could be likely from the tamarind source. An earlier study [Subramanian, Dhana Ramalakshmi 2010] indicated the reason for sorption of fluoride could also be through hydrogen bonding in tartaric acid.

\section{Fourier Transform Infra-Red characterization}

Comparison of the peaks obtained by FTIR Analysis (Figure 3) over the Mid-IR Region from $4500-400 \mathrm{~cm}^{-1}$ reveal peaks corresponding to $\mathrm{C}-\mathrm{H}$ wag and $\mathrm{C}=\mathrm{O}$ stretch. The peak of interest here is between 1300 and $1150 \mathrm{~cm}^{-1}$ corresponds to $\mathrm{C}-\mathrm{H}$ wag. It can be seen in Figure 3 that the peak reduces in the spent beads (Tamarind seed immobilized sodium alginate beads used for fluoride removal) compared to the unused loaded beads, possibly due to the replacement of $\mathrm{H}$ with $\mathrm{F}^{-}$due to fluoride removal by hydrogen bonding to tartaric acid.

\section{Brunauer-Emmett-Teller pore size analysis}

BET analysis gave the exact pore size distribution, pore radius and micropore volume. The pore radius was found to be $15.8 \mathrm{~A}^{\circ}$ and the micropore volume was found to be $0.005 \mathrm{cc}^{-1}$. Figure 4 shows the DA Plot obtained by nitrogen multilayer adsorption.

\section{Removal of fluoride by batch tests}

It was determined that the average concentration of fluoride in the field water samples collected from fluoride contaminated areas was 1.996 $\mathrm{mg}^{-1} \mathrm{l}^{-1}$, which is well above the permissible limits of $1.5 \mathrm{mg}^{-1} \mathrm{l}^{-1}$ [WHO 1984]. Hence, a synthetic fluoride standard of $2 \mathrm{mg}^{-1}{ }^{-1}$ was used. $2 \mathrm{~g}$ of the bio-sorbent in $200 \mathrm{ml}$ of synthetic fluoride water sample containing $2 \mathrm{mg}^{-1} \mathrm{l}^{-1}$ was agitated in a rotary shaker $(120 \mathrm{rpm})$. After 2 hours of contact time, the filtrate contained $0.2486 \mathrm{mg}^{-1} 1^{-1}$ of fluoride which indicates $87 \%$ removal efficacy.

\section{Factors influencing removal of fluoride by column studies}

Three factors i.e. flow rate, retention time and the number of runs were studied for optimizing the use of the absorbent for de-fluoridation purposes.

\section{Effect of flow rate and number of runs on de- fluoridation}

The effect of flow rate is usually an important factor that controls absorption. In this study, it was controlled using a peristaltic pump. Fluoride removal efficacy was tested with three different flow rates i.e., $70 \mathrm{ml}^{-h^{-1}}, 90 \mathrm{ml}^{-1} \mathrm{~h}^{-1}$ and $110 \mathrm{ml}^{-h^{-1}}$. $70 \mathrm{ml}^{-1}$ gave the best fluoride removal rates reducing levels to $0.7-0.5 \mathrm{mg}^{-1} \mathrm{l}^{-1}$ (Figure 5a). Slower flow rates would be explored in the future study.

Multiple runs of the filtrate were tested for each of the flow rates. The first run brought down

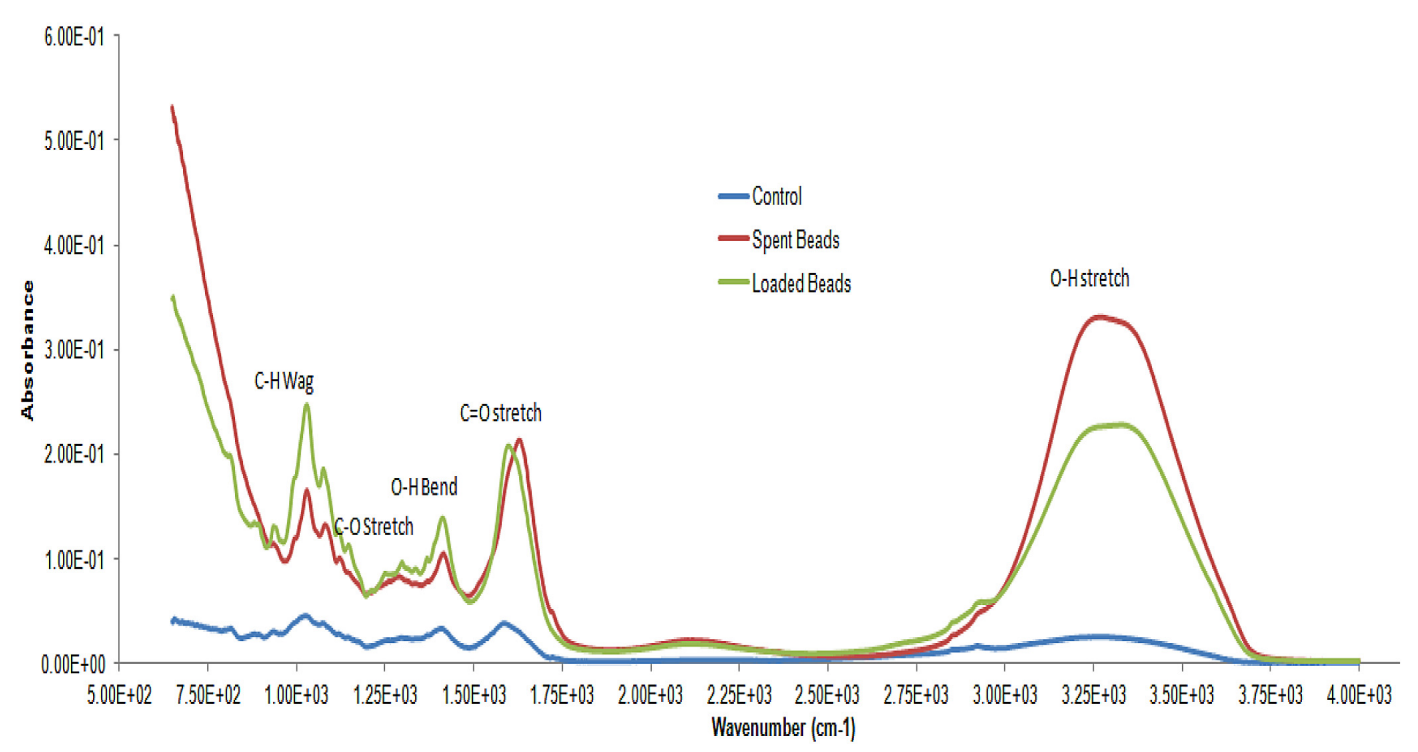

Figure 3. FTIR spectrum in the Mid - IR Region 


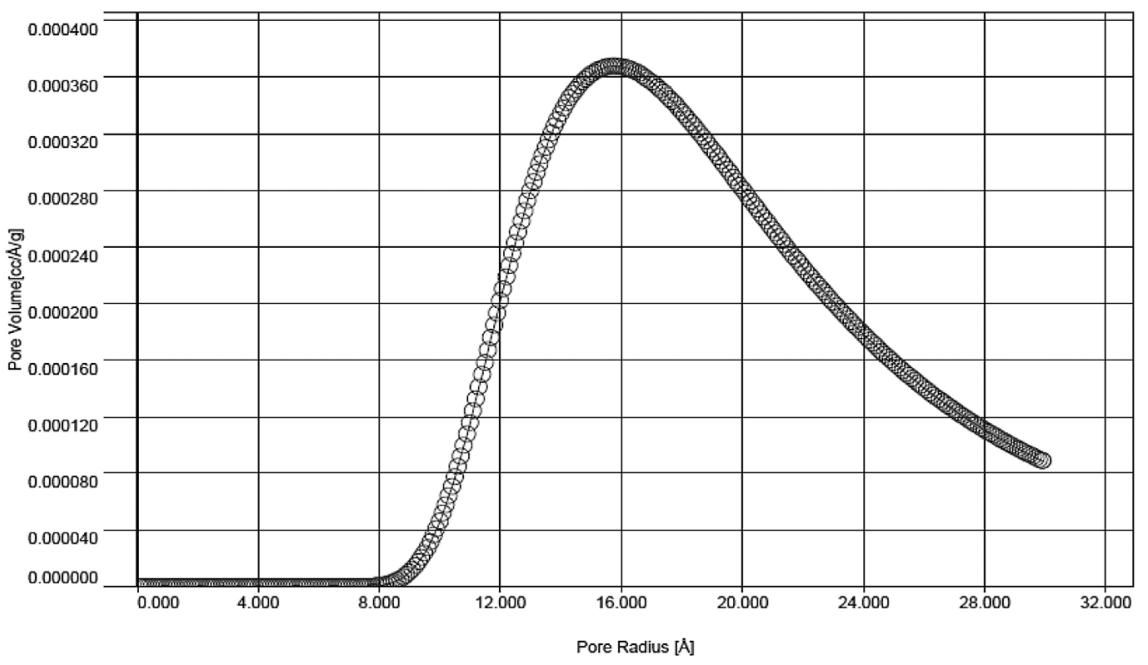

Figure 4. DA plot obtained from nitrogen multilayer adsorption
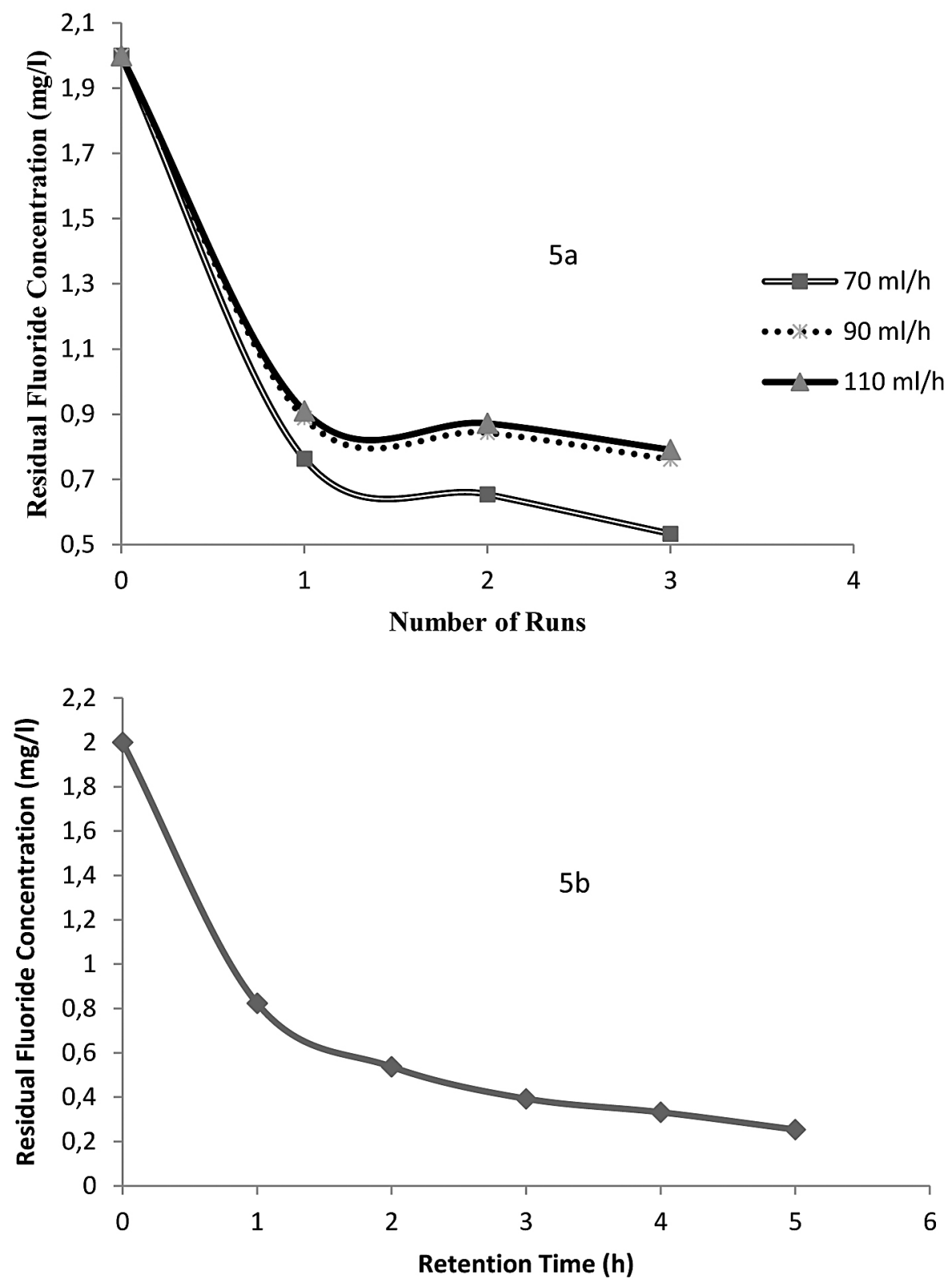

Figure 5. a - effect of flow rate and number of runs, $b$ - effect of retention time on efficacy of fluoride removal 
the fluoride concentration from $2 \mathrm{mg}^{-1} \mathrm{l}^{-1}$ to 0.72 $\mathrm{mg}^{-1} \mathrm{l}^{-1}$. The effect of subsequent runs was studied. It was found that the fluoride removal increased with each run (Figure 5a). The third run filtrate was found to have the least concentration of fluoride in all the three flow rates.

\section{Effect of retention time}

Retention time is another factor that affects adsorption of any chemical species. Greater retention time allows more adsorption to take place. Figure $5 \mathrm{~b}$ indicates that in the first one hour, the fluoride level dropped down drastically from $2 \mathrm{mg}^{-1} \mathrm{l}^{-1}$ to $0.82 \mathrm{mg}^{-1} \mathrm{l}^{-1}$ allowable ranges of fluoride concentration. Further hours of retention in the bio-sorbent seems to enable removal of more fluoride from the water as can be seen from Figure 5b.

\section{CONCLUSIONS}

Tamarind seed has been extensively used for fluoride adsorption purposes. This study improved the handling, storage and transport of the adsorbent by incorporating it in an alginate matrix. The study clearly indicated the potential of tamarind seed powder as a bio-sorbent for the removal of fluoride. The immobilization of tamarind seed powder in sodium alginate gel beads seems to have a synergistic effect on de-fluoridation. Moreover, it prevents colouration of water due to the sorbent. EDX of the sorbent revealed the presence of organics, magnesium and aluminium, which may be responsible for de-fluoridation. The flow rate, retention time and the number of runs were factors that influenced the fluoride removal efficacy of the system.

\section{Acknowledgement}

The authors wish to thank the Director, CSIRNEERI and the Director CSIR-SERC for their support and permission to carry out this research work in Chennai Zonal Laboratory of CSIRNEERI.

\section{REFERENCES}

1. World Health Organization (WHO) 1984. Guidelines for drinking water quality. Geneva, Switzerland.

2. Felsenfeld A.J., Robert M.A. 1991. A report of fluorosis in the United States secondary to drink- ing well water. J. American Medical Association, 265(4), 486-488.

3. World Health Organization (WHO) 2002. Environmental health criteria 227 fluorides. Geneva, Switzerland.

4. Kudesia V.P. 2003. Environmental chemistry. Pragati Prakashan, Meerut, $2^{\text {nd }}$ ed., 145-148.

5. Ceopalan C. 2003. The changing epidemiology of malnutrition in developing society. Current Science, 77, pp. 1257.

6. Jamodei A.V., Sapkal V.S., Jamode V.S. 2004. Defluoridation of water using inexpensive adsorbents. J. Ind. Inst. Sci., 84, 163-171.

7. Gonzales C., Hotokezaka H., Karadeniz E.I., Miyazaki T., Kobayashi E., Darendeliler M.A., Yoshida N. 2004. Effects of fluoride intake on orthodontic tooth movement and orthodontically induced root respiration. Am. J. Ortho. Dentofacial Orthop., 139, 196-205.

8. Solangi I.B., Memon S., Bhanger M.I. 2009. Removal of fluoride from aqueous environment by modified amberlite resin. J. Hazardous. Materials, 171, 815-819.

9. Meenakshi, Maheshwari R.C. 2006. Fluoride in drinking water and its removal. J. Hazardous. Materials, 137(1), 456-463.

10. Ige O.O., Umoru L.E., Aribo S. 2012. Natural products: A minefield of biomaterials. ISRN Materials Science, Article ID 983062, doi: 10.5402/ 2012/983062.

11. Subramanian E., Dhana Ramalakshmi R. 2010. Pristine, purified and polyaniline - coated taamrind seed (Tamarindus indica) biomaterial powders for defluoridation: Synergism and enhancement in fluoride adsorption by polyaniline coating. J. Sc. Ind. Res., 69, 621-628.

12. Mondal N.K., Bhaumik R., Baur T., Das B., Roy P., Datta J.K. 2012. Studies on defluoridation of water by tea ash: An unconventional biosorbent. Chem. Sc. Trans., 1(2), 239-256.

13. Mishra A. 2013. Tamarind seed xyloglucan: A food hydrocolloid for water remediation. J. Biobased. Mater. Bioenerg., 7, 12-18.

14. American Public Health Association (APHA / AWWA / WEF). 2005. Standard methods for the examination of water and wastewater. $21^{\text {st }}$ ed. Washington, DC.

15. Handa B.K. 1975. Geochemistry and genesis of fluoride containing groundwater in India. Ground water, 13, 278-281.

16. Brindha K., Rajesh R., Murugan R., Elango R. 2011. Fluoride contamination in groundwater in parts of Nalgonda District, Andhra Pradesh, India. Environ. Monit. Assess., 172(1-4), 481-492. 